\title{
Using logic models to enhance the methodological quality of primary health-care interventions: guidance from an intervention to promote nutrition care by general practitioners and practice nurses
}

\author{
Lauren Ball $^{\mathrm{A}, \mathrm{H}}$, Dianne Ball ${ }^{\mathrm{B}}$, Michael Leveritt ${ }^{\mathrm{C}}$, Sumantra Ray ${ }^{\mathrm{D}}$, Clare Collins ${ }^{\mathrm{E}}$, Elizabeth \\ Patterson $^{\mathrm{F}}$, Gina Ambrosini ${ }^{\mathrm{G}}$, Patricia Lee ${ }^{\mathrm{A}}$ and Wendy Chaboyer ${ }^{\mathrm{A}}$ \\ ${ }^{\mathrm{A}}$ Menzies Health Institute Queensland, Griffith University, Gold Coast, Qld, 4222 Australia.

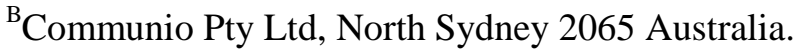

${ }^{\mathrm{C}}$ School of Human Movement and Nutrition Sciences, The University of Queensland, Brisbane, 4072 Qld, Australia.

${ }^{\mathrm{D}}$ The Need for Nutrition Education/Innovation Programme, The University of Cambridge, Cambridge, UK.

${ }^{E}$ Priority Research Centre for Physical Activity and Nutrition, The University of Newcastle, NSW, 2308, Australia.

${ }^{\mathrm{F}}$ Faculty of Medicine, Dentistry and Health Sciences, The University of Melbourne, Vic., 3052, Australia.

${ }^{\mathrm{G}}$ School of Population Health, The University of Western Australia, Crawley, WA, 6009, Australia.

${ }^{\mathrm{H}}$ Corresponding author. Email: 1.ball@griffith.edu.au

The methodological designs underpinning many primary health-care interventions are not rigorous. Logic models can be used to support intervention planning, implementation and evaluation in the primary health-care setting. Logic models provide a systematic and visual way of facilitating shared understanding of the rationale for the intervention, the planned activities, expected outcomes, evaluation strategy and required resources. This article provides guidance for primary health-care practitioners and researchers on the use of logic models for enhancing methodological rigour of interventions. The article outlines the recommended steps in developing a logic model using the 'NutriCare' intervention as an example. The 'NutriCare' intervention is based in the Australian primary health-care setting and promotes nutrition care by general practitioners and practice nurses. The recommended approach involves canvassing the views of all stakeholders who have valuable and informed opinions about the planned project. The following four targeted, iterative steps are recommended: (1) confirm situation, intervention aim and target population; (2) document expected outcomes and outputs of the intervention; (3) identify and describe assumptions, external factors and inputs; and (4) confirm intervention components. Over a period of 2 months, three primary health-care researchers and one health-services consultant led the collaborative development of the 'NutriCare' logic model. Primary health-care practitioners and researchers are encouraged to develop a logic model when planning interventions to maximise the methodological rigour of studies, confirm that data required to answer the question are captured and ensure that the intervention meets the project goals. 


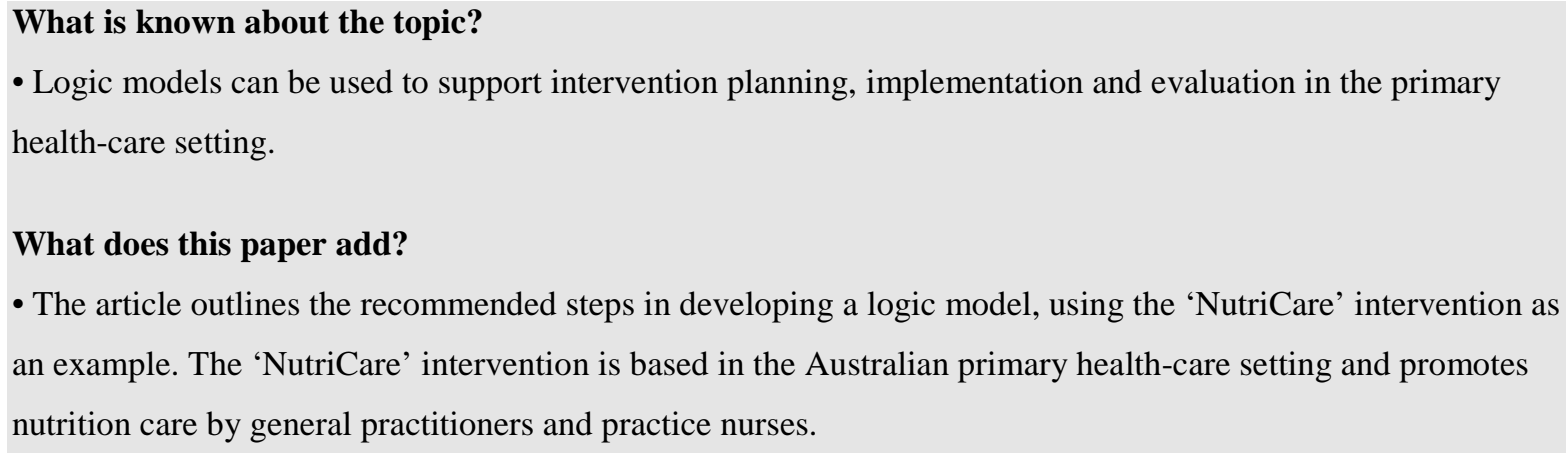

\section{What does this paper add?}

- The article outlines the recommended steps in developing a logic model, using the 'NutriCare' intervention as an example. The 'NutriCare' intervention is based in the Australian primary health-care setting and promotes nutrition care by general practitioners and practice nurses.

PY16038

Logic models for intervention planning

Additional keywords: chronic disease, general practice, intervention studies, logic model, nutritional management, nutrition therapy, primary care, research methods.

\section{Background}

Primary health-care interventions require robust methodologies to maximise the confidence of conclusions drawn from studies (Greenhalgh 2007). However, the methodological designs underpinning many primary health-care interventions are not considered rigorous (Beck et al. 2002; Jacobson and Gance-Cleveland 2011; Orrow et al. 2012; Smith et al. 2012). Two common reasons for suboptimal methodologies are the increasing emphasis on capacity-building initiatives to engage primary health-care practitioners with modest research expertise to participate in research (Friesen et al. 2014); and increasing use of multi-component, or 'complex' interventions, which requires advanced skills in methodological design and large teams (Craig et al. 2008). It is subsequently recognised that primary health-care practitioners and researchers would benefit from greater support to enhance the methodological design underpinning interventions.

Logic models provide a systematic and visual way of determining the planned research activities and expected outcomes of interventions (Arts and Humanities Research Council UK 2015). Logic models were initially used for program planning and evaluation (Wholey 1979), and are now increasingly recommended as a step in developing health-care interventions (Guttmacher et al. 2010). Although their format and scope are variable, the following six components are usually included in logic models: the situation, inputs, outputs, outcomes, assumptions and external factors (TaylorPowell and Henert 2008). Developing a logic model when planning an intervention is useful for clarifying the logic underpinning the intervention, identifying gaps in resources and in facilitating a shared understanding of the purpose of intervention among stakeholders and team members. In addition, logic models create a visual and conceptual link between the intervention and broad program goals. After implementing an intervention, logic models provide a valuable basis for formative and summative evaluations (Arts and Humanities Research Council UK 2015). Despite evidence of logic models being used to support intervention development in public health (Joly et al. 2007; Das et al. 
2014), community (Chen et al. 1999; Medeiros et al. 2005) and acute-care settings (Subirana et al.

2014), their utilisation in the primary health-care setting is less established (Humphreys et al. 2009;

Hayes et al. 2011).

The recommended approach to developing a logic model involves seeking input and examining the views of all stakeholders who have informed opinions about the planned project (Taylor-Powell and Henert 2008). In the case of primary health-care interventions, this could involve primary health-care practitioners, researchers, patients and carers, funders, commissioning services, and industry or pharmaceutical representatives. The present article aims to provide guidance to primary health-care practitioners and researchers on the use of logic models for enhancing the methodological quality of interventions within the primary-care setting. The article outlines the steps involved in developing a logic model for the primary health-care setting by using an example of a complex intervention, the 'NutriCare' intervention. The NutriCare intervention aims to support general practitioners (GPs) and practice nurses (PNs) to provide nutrition care to patients in consultations.

\section{Methods}

\section{Overview}

A team of three primary health-care researchers and one health-services consultant led the collaborative development of the NutriCare logic model over a period of 2 months. The team had diverse research experience and utilised learning resources from the University of Wisconsin Logic Model training module (Taylor-Powell and Henert 2008), the W. K. Kellogg Foundation (W. K. Kellogg Foundation 2006) and Center for Disease Control and Prevention to guide the development process (Centers for Disease Control and Prevention 2015). The team developed the logic model in an iterative, consultative manner after a review of original research, behaviour change theories used in the field of knowledge translation, informal and formal meetings to canvass feedback from funders and commissioning groups (such as Primary Health Networks), peer researchers in dietetics, nursing and medicine, as well as focus groups with patients, GPs and PNs. Focus groups were organised through the local Primary Health Network, where 20 patient representatives and 18 health professionals provided ongoing feedback to the research team regarding the logic model.

Four targeted, iterative steps were drafted and confirmed by the team, on the basis of the premise of 'working backwards to implement forwards' (Taylor-Powell and Henert 2008), and included the following: (1) confirm situation, intervention aim and target population; (2) document expected outcomes and outputs of the intervention; (3) identify and describe assumptions, external factors and inputs; and (4) confirm intervention components. 
Step 1: confirm situation, intervention aim and target population

'Situation' refers to the overall context in which a study will be implemented, as well as the key problems or issues that the study will attempt to address. The situation statement is usually placed on the left, or on the top, of the logic model to clarify the broad setting for the intervention. The aim of the intervention will determine the level of complexity required in the model, and should be aligned with the situation or context in which the intervention will occur. The aim is often placed at the top of the logic model. In many situations, more than one intervention is worthy of being conducted, and the logic model should include only the intervention aim that has been prioritised. The target population refers to the group, or type of people, that the intervention is seeking to influence. In primary healthcare research, this is usually a patient population (for example, males aged over 50 years with history of hypertension), or a health professional population (for example, GPs who work in rural locations). The target population should be specified within the intervention aim.

\section{Step 2: document expected outcomes and outputs of the intervention}

Outcomes refer to the ultimate improvements that are intended to occur as a result of an intervention, and are usually placed on the right-hand side of a logic model. The term 'outcome' is often used interchangeably with 'impact'. Ideally, short-term changes in outcomes (such as increased knowledge), and medium-term (such as change in behaviour or practice) and long-term (such as change in health status) outcomes are identified (Taylor-Powell and Henert 2008). The periods of time considered to be short-, medium- and long-term will vary for different interventions, and should, therefore, be specified and appropriate for the proposed intervention. Outputs are listed in the centre of the logic model, and refer to the activities required or tasks to be undertaken to implement an intervention, as well as specifying the stakeholders that are needed to conduct and/or engage in the activities. These activities can include meetings, training, screening, recruitment, intervention delivery, data collection, analysis, interpretation and dissemination. The participants include the potential participant pool for the intervention, as well as stakeholders who facilitate implementation of the study, such as researchers and assistants, health-care practitioners, practice support staff, primaryhealth networks, patients and carers.

\section{Step 3: identify and describe assumptions, external factors and inputs}

Assumptions refer to the beliefs about the way the intervention is anticipated to work, and these are usually listed on the left-hand side of the logic model. Ideally, the assumptions should be based on evidence and behaviour-change theory, and can include beliefs about the situation, resources, environment or participants (Taylor-Powell and Henert 2008). The logic model should clearly articulate as many implicit assumptions about the intervention as possible. Development of the logic model provides opportunities for the intervention developers to discuss the assumptions in detail. External factors refer to the environment in which the intervention will be delivered. They are 
generally outside of the control of the intervention team, but may influence the intervention outcomes. External factors are also listed on the left-hand side, and can include the political climate, health policy climate, cultural climate, media influence or changing priorities within a setting. Inputs refer to the resources required to adequately implement the intervention. The resources can include personnel, funding, materials, equipment, partnerships and technology. Inputs are regarded as a resource area that is most likely to hinder intervention implementation as anticipated. The intervention team is encouraged to continually revisit the inputs after implementation to identify gaps as they arise (Taylor-Powell and Henert 2008).

\section{Step 4: confirm intervention components}

After all sections of the logic model have been drafted, greater inspection of the intervention components is recommended. This is usually identified on the logic map as part of the outputs (W. K. Kellogg Foundation 2006). The components of a complex intervention are integral to ensuring that the outputs produce the desired outcomes of the intervention. This final step should confirm the theoretical basis underpinning the intervention components. For example, the behaviour changetechnique matrix (Cane et al. 2012) has been successfully used in intervention studies in the Australian primary health-care setting (Mazza and Chapman 2010; McKenzie et al. 2010). The purpose of the matrix is to facilitate the development of theory-based interventions that have clear causal pathways between intervention components and barriers and facilitators to health-professional behaviours.

\section{Results}

The resulting logic model developed for the 'NutriCare' intervention is shown in Fig. 1 and highlights the four steps used to develop the logic model.

\section{Step 1: confirm situation, intervention aim and target population}

\section{Situation}

The primary health-care setting was identified as an ideal environment for initiatives that facilitate patients to improve their dietary behaviour (Australian Government 2013). Nearly all adults are at risk of developing a chronic disease as a result of poor dietary behaviour (Imamura et al. 2015), making dietary behaviour the most common modifiable risk factor for chronic disease (Lim et al. 2012). Practice guidelines recommend that GPs and PNs advocate about the importance of healthy eating and drinking behaviour at every appropriate opportunity when in consultations with adult patients (Royal Australian College of General Practitioners 2015). However, GPs and PNs experience many barriers to the inclusion of nutrition care in consultations, and only discuss nutrition in $~ 7 \%$ of all consultations (Britt et al. 2015). As a result, the rate that GPs and PNs discuss nutrition in consultations is considered suboptimal. 


\section{Intervention aim}

The most important aim in this situation was identified as an intervention that reduces the barriers to GPs and PNs incorporating nutrition care in consultations. Achieving this aim will increase the frequency that GPs and PNs provide nutrition care to patients, which will subsequently support improved dietary behaviours of patients.

\section{Target population}

The most appropriate target population was identified as GPs and PNs across Australia. The rationale for the target population included the following: (1) 90\% of Australian adults consult a GP or PN at least once per year; (2) over 60\% of Australian general-practice clinics hire a PN to support their primary health-care services (Australian Practice Nurses Association, see http://www.apna.asn.au, accessed); and (3) there is recognised potential for nutrition care by GPs and PNs to improve patients’ dietary behaviours (Ball et al. 2015).

\section{Step 2: document expected outcomes and outputs of the intervention}

\section{Outcomes}

The desired long-term outcomes were identified as improvements in the dietary behaviour of adult patients as measured by the Australian eating survey, as well as improved biomarkers of lifestylerelated chronic disease 12 months after the intervention is implemented. To facilitate the long-term outcomes, the medium-term outcome was defined as an increased frequency of providing nutrition care within consultations with adult patients 3 months after the intervention is implemented, so that more patients with dietary risk factors for chronic disease receive nutrition care when clinically appropriate. This outcome is a measure of clinical activity and is a process measure. To facilitate the medium-term outcome, short-term outcomes were defined as GPs and PNs (1) experiencing fewer barriers to nutrition care, and (2) feeling more confident and competent at incorporating nutrition care in consultations after the intervention has been implemented.

\section{Outputs}

The desired outputs of the NutriCare intervention were identified as (1) collaborative communication with stakeholders to identify potential participants for the study, (2) delivery of the intervention and (3) collection and review of data. These outputs will require participation from GPs and PNs, researchers, practice managers, support staff and primary-health networks.

Step 3: identify and describe assumptions, external factors and inputs

\section{Assumptions}

Three assumptions of the NutriCare intervention were identified and supported by behaviourchange theory and literature. The assumptions were that (1) the intervention will adequately produce the desired outcomes, (2) patients with dietary risk factors for chronic disease will be receptive to 
receiving nutrition care from GPs and PNs within consultations (Hegney et al. 2013; Ball et al. 2014) and (3) the resultant nutrition care provided by GPs and PNs will achieve the overall goal of supporting the adoption of healthy dietary behaviour in adults at risk of chronic disease (Ball et al. 2013, 2015).

\section{External factors}

Two external factors were deemed as most relevant to the NutriCare intervention, and reflected ongoing changes to the policy and funding structure of the Australian primary health-care setting.

\section{Inputs}

The inputs of the NutriCare intervention were identified as appropriately skilled research members, sufficient funding, confirmed methodology and data-collection procedures, as well as fully developed and tested intervention components.

\section{Step 4: confirm intervention components}

The four components of the NutriCare intervention were developed using the behaviour changetechnique matrix (Cane et al. 2012), and are outlined in Table 1. The table explains the causal link between the intervention components and anticipated outcomes. Each intervention component targets one of the following three most commonly reported barriers to GPs and PNs incorporating nutrition care in consultations: (1) low self-efficacy (Levine et al. 1993; Kushner 1995; Hiddink et al. 1997;

Cass et al. 2014; Martin et al. 2014); (2) lack of nutrition knowledge (Levine et al. 1993; Hopper and Barker 1995; Kushner 1995; Ball et al. 2010; Cass et al. 2014; Martin et al. 2014); and (3) limited time in consultations (Kushner 1995; Hiddink et al. 1997; Ball et al. 2010; Wynn et al. 2010). For each targeted barrier, a description of the relevant behaviour domain from the behaviour changetechnique matrix is outlined. The table also shows the (1) type, (2) mode and (3) content of the intervention component that will target the barrier and the mechanism of action, justifying how the component will achieve success in reducing the barrier.

\section{Discussion}

This article provides guidance on the use of logic models for enhancing the methodological quality of interventions in primary health care. Logic models can be developed for interventions targeting different levels of change (W. K. Kellogg Foundation 2006; Taylor-Powell and Henert 2008; Centers for Disease Control and Prevention 2015). For example, logic models can be developed at a 'macro' level to address broad programs of research, at a 'meso' level for studies within a broad program of research, or at a 'micro' level for targeted, one-off studies (Taylor-Powell and Henert 2008). The NutriCare logic model is an example of a meso-level project, because it is one study within a program of research that supports the optimal provision of nutrition care to patients with dietary risk factors for chronic disease who attend primary-care consultations. Therefore, the NutriCare intervention will 
contribute to the recognised need for the primary health-care setting to support patients to have healthy lifestyle behaviours, including healthy dietary behaviours (Australian Government 2013).

The benefits and challenges of using logic models have been previously documented (Kaplan and Garrett 2005). Three benefits are particularly relevant to the primary health-care setting; namely, logic models help (1) build consensus through collaboration with a variety of stakeholders, (2) strengthen the design of interventions by clarifying underlying assumptions and addressing barriers and facilitators for implementation and 3) demonstrate how primary health-care interventions can influence health outcomes at a population level. However, the greatest challenge of developing a logic model is the time required to engage in discussions with stakeholders. This challenge is particularly relevant in primary health care, where a lack of time is inherently experienced as a barrier to intervention planning and overall research capacity (Farmer and Weston 2002). Given that the effectiveness and utility of a logic model is dependent on the engagement and discussion of stakeholders (Kaplan and Garrett 2005; Taylor-Powell and Henert 2008), it is essential that steps are taken to facilitate open communication among stakeholders in this setting.

The UK Medical Research Council recommends the use of theory in the development of multifaceted interventions (Moore et al. 2015). Furthermore, logic models are recommended to be developed during the planning stage of an intervention (Guttmacher et al. 2010). Unlike decisionanalysis tools, logic models are also useful during the implementation and evaluation stages of research (Arts and Humanities Research Council UK 2015). For example, during the implementation stage of research, logic models can be used as a reminder of the aims, activities and processes of a project, and facilitate continuous improvement. The model can be used as a basis for formative evaluation, and can strengthen communication and commitment between the intervention team and stakeholders. After implementation has been completed, logic models contribute to the evaluation of the effectiveness of the intervention. The logic model can be used as a basis for summative evaluation, where performance indicators are assessed against pre-determined targets. These actions assist in strengthening the link among the intervention, recommendations and policy directives (Arts and Humanities Research Council UK 2015).

It is important to acknowledge that the benefit of using a logic model cannot be rigorously tested through implementation research because of the unique context of workplaces and variable intervention designs. The logic model for the 'NutriCare' intervention is one example of a logic model in use at a pre-implementation time point. The 'NutriCare' logic model will be amended after a pilot of the intervention occurs and again after implementation. In addition, the logic model will be used as the foundation for conducting the process and impact evaluations. The depiction of the logic model in graphic form can vary depending on the creativity and perspective of the developers (W. K. Kellogg Foundation 2006). Updating the logic model by reflecting on the progress as the intervention becomes established is an example of action learning (Casey 2007). Continually reflecting on the 
logic model is important in increasing the likelihood that positive outcomes of the intervention can be sustained through translation to ongoing health-care practices.

In conclusion, logic models help enhance the methodological rigour of interventions through engagement with stakeholders. The development process of the 'NutriCare' logic model can act as a guide when planning interventions. Researchers and primary health-care professionals are encouraged to develop logic models when planning, implementing and evaluating interventions.

\section{Funding}

L. Ball is funded by the Australian National Health and Medical Research Council through an Early Career Research Fellowship.

\section{Conflicts of interest}

None declared.

\section{Acknowledgements}

xxx The authors would like to acknowledge the stakeholders who provided input to the development of the logic model.

\section{References}

$<$ bok>Arts and Humanities Research Council UK (2015) 'Logic Models for Programme Planning and

Evaluation.' (AHRC: Swindon, UK) $</$ bok $>$

<other>Australian Government (2013) 'National Primary Health Care Strategic Framework.' (Commonwealth of Australia: Canberra) $</$ other $>$

<jrn>Ball L, Hughes R, Leveritt M (2010) Nutrition in general practice: role and workforce preparation expectations of medical educators. Australian Journal of Primary Health 16, 304-310. doi:10.1071/PY10014</jrn>

$<$ jrn>Ball L, Johnson C, Desbrow B, et al. (2013) General practitioners can offer effective nutrition care to patients with lifestyle related chronic disease: a systematic review. Journal of Primary Health Care 5, 5969. $<$ jirn>

$<$ jrn>Ball L, Desbrow B, Leveritt M (2014) An exploration of individuals' preferences for nutrition care from Australian primary care health professionals. Australian Journal of Primary Health 20, 113-120. doi:10.1071/PY12127 </jrn>

$<$ jrn>Ball L, Leveritt M, Cass S, et al. (2015) Effect of nutrition care provided by primary health professionals on adults’ dietary behaviours: a systematic review. Family Practice 32, 605-617.</jrn>

$<$ jrn>Beck RS, Daughtridge R, Sloane PD (2002) Physician-patient communication in the primary care office: a systematic review. The Journal of the American Board of Family Practice 15, 25-38.</jrn>

<bok>Britt H, Mitter G, Henderson J, et al. (2015) ‘General practice activity in Australia 2014-15. General

Practice Series Number 34.' (Canberra)</bok $>$ 
$<$ jrn>Cane J, O'Connor D, Michie S (2012) Validation of the theoretical domains framework for use in behaviour change and implementation research. Implementation Science; IS 7, 37. doi:10.1186/1748-5908-7$\underline{\underline{37}</ \mathrm{jrn}>}$

$<$ jrn>Casey D (2007) Using action research to change health-promoting practice. Nursing \& Health Sciences $\mathbf{9}$, 5-13. doi:10.1111/j.1442-2018.2007.00297.x</jrn>

$<$ jrn>Cass S, Ball L, Leveritt M (2014) Australian practice nurses' perceptions of their role and competency to provide nutrition care to patients living with chronic disease. Australian Journal of Primary Health 20, 203208. doi:10.1071/PY12118</jrn>

$<$ eref>Centers for Disease Control and Prevention (2015) Evaluation resources: manuals/assistance with specific evaluation steps - logic models. Available at http://www.cdc.gov/eval/resources/ [Verified day month year] $</$ eref $>$

$<$ jrn>Chen WW, Cato BM, Rainford N (1999) Using a logic model to plan and evaluate a community intervention program: a case study. International Quarterly of Community Health Education 18, 449-458. doi:10.2190/JDNM-MNPB-9P25-17CQ</jrn>

$<$ jrn>Craig, P., Dieppe, P., Macintyre, S., et al. (2008). Developing and evaluating complex interventions: the new Medical Research Council guidance. BMJ, 337, a1655. doi:10.1136/bmj.a1655</jrn>

$<$ jrn>Das BM, Petruzzello SJ, Ryan KE (2014) Development of a logic model for a physical activity-based employee wellness program for mass transit workers. Preventing Chronic Disease 11, 140124. doi:10.5888/pcd11.140124</jrn>

$<j r n>$ Farmer E, Weston K (2002) A conceptual model for capacity building in Australian primary health care research. Australian Family Physician 31, 1139-1142.</jrn>

$<$ jrn>Friesen EL, Comino EJ, Reath J, et al. (2014) Building research capacity in south-west Sydney through a Primary and Community Health Research Unit. Australian Journal of Primary Health 20, 4-8. doi:10.1071/PY12081</jrn>

$<$ bok>Greenhalgh, T. (2007) 'Primary Health Care Theory and Practice.' (Blackwell Publishing \& BMJ Books) $</$ bok $>$

$<$ bok>Guttmacher S, Kelly P, Ruiz-Janecko Y (2010) ‘Community-Based Health Interventions.' (Wiley)</bok>

$<$ jrn>Hayes H, Parchman ML, Howard R (2011) A logic model framework for evaluation and planning in a primary care practice-based research network (PBRN). Journal of the American Board of Family Medicine 24, 576-582. doi:10.3122/jabfm.2011.05.110043</jrn>

$<$ jrn>Hegney DG, Patterson E, Eley DS, et al. (2013) The feasibility, acceptability and sustainability of nurseled chronic disease management in Australian general practice: the perspectives of key stakeholders. International Journal of Nursing Practice 19, 54-59. doi:10.1111/ijn.12027</jrn>

$<$ jrn>Hiddink G, Hautvast H, Van Woerkum C, et al. (1997) Driving forces for and barriers to nutrition guidance practice of Dutch primary care physicians. Journal of Nutrition Education 29, 36-41. doi:10.1016/S0022-3182(97)70144-4</jrn> 
<jrn>Hopper D, Barker M (1995) Dietary advice, nutrition knowledge and attitudes towards nutrition in primary care. Journal of Human Nutrition and Dietetics 8, 279-286. doi:10.1111/j.1365-277X.1995.tb00321.x</jrn>

<bok>Humphreys J, Wakerman J, Kuipers P (2009) 'Improving Workforce Retention: Developing an Integrated Logic Model to Maximise Sustainability of Small Rural and Remote Health Care Services.' (Australian Primary Health Care Research Institute: Canberra)</bok>

<jrn>Imamura F, Micha R, Khatibzadeh S, et al. (2015) Dietary quality among men and women in 187 countries in 1990 and 2010: a systematic assessment. The Lancet. Global Health 3, e132-e142. doi:10.1016/S2214-109X(14)70381-X</jrn>

$<$ jrn>Jacobson D, Gance-Cleveland B (2011) A systematic review of primary healthcare provider education and training using the chronic care model for childhood obesity. Obesity Reviews 12, e244-e256. doi:10.1111/j.1467-789X.2010.00789.x</jrn>

$<$ jrn>Joly BM, Polyak G, Davis MV, et al. (2007) Linking accreditation and public health outcomes: a logic model approach. Journal of Public Health Management and Practice 13, 349-356. doi:10.1097/01.PHH.0000278027.56820.7e </jrn>

$<$ jrn>Kaplan S, Garrett K (2005) The use of logic models by community-based initiatives. Evaluation and Program Planning 28, 167-172. doi:10.1016/j.evalprogplan.2004.09.002</jrn>

$<$ jrn>Kushner R (1995) Barriers to providing nutrition counselling by physicians. Preventive Medicine 24, 546552. doi:10.1006/pmed.1995.1087</jrn>

$<j r n>$ Levine B, Wigren M, Chapman D, et al. (1993) A national survey of attitudes and practices of primarycare physicians relating to nutrition: strategies for enhancing the use of clinical nutrition in medical education. The American Journal of Clinical Nutrition 57, 115-119.</jrn>

$<j r n>$ Lim SS, Vos T, Flaxman AD, et al. (2012) A comparative risk assessment of burden of disease and injury attributable to 67 risk factors and risk factor clusters in 21 regions, 1990-2010: a systematic analysis for the global burden of disease study 2010. Lancet 380, 2224-2260. doi:10.1016/S0140-6736(12)61766-8</jrn>

$<$ jrn>Martin L, Leveritt M, Desbrow B, et al. (2014) The self-perceived knowledge, skills and attitudes of Australian practice nurses in providing nutrition care to patients with chronic disease. Family Practice 31, 201-208. doi:10.1093/fampra/cmt070</jrn>

$<$ jrn>Mazza D, Chapman A (2010) Improving the uptake of preconception care and periconceptional folate supplementation: what do women think? BMC Public Health 10, 786. doi:10.1186/1471-2458-10-786</jrn>

$<\mathrm{jrn}>$ McKenzie JE, O’Connor DA, Page MJ, et al. (2010) Improving the care for people with acute low-back pain by allied health professionals (the ALIGN trial): a cluster randomised trial protocol. Implementation Science; IS 5, 86. doi:10.1186/1748-5908-5-86</jrn>

$<$ jrn>Medeiros LC, Butkus SN, Chipman H, et al. (2005) A logic model framework for community nutrition education. Journal of Nutrition Education and Behavior 37, 197-202. doi:10.1016/S1499-4046(06)60246$\underline{\underline{7}}<$ jjrn $>$ 
$<$ jrn>Moore G, Audrey S, et al. (2015). Process evaluation of complex interventions: Medical Research Council guidance. British Medical Journal 350, h1258. doi:10.1136/bmj.h1258</jrn>

$<$ jrn $>$ Orrow G, Kinmonth AL, Sanderson S, et al. (2012) Effectiveness of physical activity promotion based in primary care: systematic review and meta-analysis of randomised controlled trials. BMJ (Clinical Research Ed.) 344, e1389. doi:10.1136/bmj.e1389</jrn>

$<$ bok>Royal Australian College of General Practitioners (2015) 'Smoking, Nutrition, Alcohol and Physical Activity (SNAP): a Population Health Guide to Behavioural Risk Factors in General Practice’, 2nd edn. (RACGP: Melbourne)</bok>

$<$ jrn>Smith SM, Soubhi H, Fortin M, et al. (2012) Managing patients with multimorbidity: systematic review of interventions in primary care and community settings. BMJ (Clinical Research Ed.) 345, e5205.</jrn>

$<$ jrn>Subirana M, Long A, Greenhalgh J, et al. (2014) A realist logic model of the links between nurse staffing and the outcomes of nursing. Journal of Research in Nursing 19, 8-23.

doi:10.1177/1744987113481782</jrn>

$<$ bok>Taylor-Powell E, Henert E (2008) ‘Developing a Logic Model: Teaching and training Guide.' (University of Wisconsin: Madison, WI) $<$ /bok $>$

$<$ bok>Wholey J (1979) ‘Evaluation: Promise and Performance.' (Urban Institute)</bok>

$<$ bok>W.K. Kellogg Foundation (2006) ‘Logic Model Development Guidance.’ (W. K. Kellogg Foundation

General: MO)</bok>

Received 19 March 2016, accepted 1 June 2016 
Table 1. Components of the NutriCare intervention

GP, general practitioner; PN, practice nurse

\begin{tabular}{|c|c|c|c|c|}
\hline $\begin{array}{l}\text { Compo } \\
\text { nent }\end{array}$ & $\begin{array}{l}\text { Targeted } \\
\text { barrier }\end{array}$ & TDF domain and description & Component of intervention & Mechanism of action \\
\hline 1 & $\begin{array}{l}\text { Low self- } \\
\text { efficacy of } \\
\text { GP/PN }\end{array}$ & $\begin{array}{l}\text { Beliefs about capabilities: acceptance } \\
\text { of the truth, reality, or validity about } \\
\text { an ability talent or facility that a } \\
\text { person can put to constructive use }\end{array}$ & $\begin{array}{l}\text { Type: modelling } \\
\text { Mode: desk quotes } \\
\text { Content: pre-collected excerpts from GPs and PNs } \\
\text { who feel confident in briefly providing nutrition } \\
\text { care }\end{array}$ & $\begin{array}{l}\text { The quotes will act a passive examples for the } \\
\text { types of questions and statements that can be } \\
\text { used to incorporate nutrition care into } \\
\text { consultations }\end{array}$ \\
\hline 2 & $\begin{array}{l}\text { Lack of } \\
\text { nutrition } \\
\text { knowledge of } \\
\text { GP/PN }\end{array}$ & $\begin{array}{l}\text { Knowledge: awareness of the } \\
\text { existence of something }\end{array}$ & $\begin{array}{l}\text { Type: education } \\
\text { Mode: fact sheet (electronic and paper-based) } \\
\text { Content: the Australian Dietary Guidelines, } \\
\text { including recommended servings of each food } \\
\text { group and serving sizes }\end{array}$ & $\begin{array}{l}\text { The fact sheet will increase participants' } \\
\text { nutrition knowledge by providing the } \\
\text { foundational nutrition information required to } \\
\text { provide nutrition care to patients }\end{array}$ \\
\hline 3 & $\begin{array}{l}\text { Limited time } \\
\text { of GP/PN }\end{array}$ & $\begin{array}{l}\text { Environmental context: any } \\
\text { circumstance of a person's situation } \\
\text { or environment that discourages or } \\
\text { encourages behaviour }\end{array}$ & $\begin{array}{l}\text { Type: persuasion } \\
\text { Mode: 10-min discussion with a respected peer } \\
\text { GP/PN 'nutrition champion' } \\
\text { Content: the discussion outlines the potential } \\
\text { impact that brief nutrition care can have on } \\
\text { patients' dietary behaviours and encourage brief } \\
\text { advocacy statements in consultations }\end{array}$ & $\begin{array}{l}\text { The discussion will use communication to } \\
\text { induce positive feelings and stimulate action } \\
\text { about including brief nutrition care in } \\
\text { consultations }\end{array}$ \\
\hline 4 & $\begin{array}{l}\text { Low priority } \\
\text { of GP/PN }\end{array}$ & $\begin{array}{l}\text { Intentions: a conscious decision to } \\
\text { perform a behaviour or a resolve to } \\
\text { act in a certain way }\end{array}$ & $\begin{array}{l}\text { Type: environmental restructuring } \\
\text { Mode: on-screen prompts on patient management } \\
\text { system } \\
\text { Content: the prompt will encourage GPs/PNs to } \\
\text { advocate about the importance of nutrition before } \\
\text { concluding the consultation }\end{array}$ & $\begin{array}{l}\text { The prompt will remind the GP/PN about } \\
\text { including nutrition care in the consultation }\end{array}$ \\
\hline
\end{tabular}

Fig. 1. Logic model of the NutriCare intervention, and steps used to develop the model. GP, general practitioner; m, months; and PN, practice nurse. 